\title{
Pulmonary scar carcinoma in South Africa
}

\author{
N Jenkins, MB ChB; E M Irusen, MB ChB, FCP (SA), PhD, FCCP; C F N Koegelenberg, MB ChB, MMed (Int), FCP (SA), \\ FRCP (UK), Cert Pulm (SA), PhD \\ Division of Pulmonology, Department of Medicine, Faculty of Medicine and Health Sciences, Stellenbosch University and Tygerberg \\ Academic Hospital, Cape Town, South Africa
}

Corresponding author: C F N Koegelenberg (coeniefn@sun.ac.za)

\begin{abstract}
Background. The association between lung scarring and the subsequent development of cancer remains controversial. South Africa has one of the highest incidences of tuberculosis in the world, and resultant scarring may predispose to malignancy. The country also carries a very high burden of smoking and smoking-related diseases that may be synergistic in malignant transformation.

Objective. To assess the frequency of pulmonary scarring in patients with lung cancer.

Methods. All patients with confirmed lung cancer and a staging computed tomography (CT) scan of the chest were included in this 2-year retrospective study. Pulmonary scarring was categorised according to location as present in: (i) the same lobe as the primary tumour, (ii) a different lobe of the same lung, or (iii) the contralateral lung; or (iv) as diffuse. Post-obstructive bronchiectasis and other changes secondary to cancer were considered not to represent scarring.

Results. We identified 435 cases of primary lung cancer. In total, 95 patients $(21.8 \%)$ had CT evidence of pulmonary scarring. Eighty-three of 85 patients $(97.6 \%)$ had focal scarring in the same lobe as the primary tumour. Of these, $37(43.5 \%)$ also had scarring involving a different lobe of the same lung, whereas only one $(1.2 \% ; p<0.001)$ had scarring isolated to a different lobe of the same lung. Moreover, 21 patients $(24.7 \%)$ also had scarring of the opposite lung, but only one patient $(1.2 \% ; p<0.001)$ had scarring isolated to the contralateral lung. Ten patients had diffuse scarring, caused by bronchiectasis $(n=5)$, idiopathic pulmonary fibrosis $(n=4)$ and silicosis $(n=1)$.

Conclusion. At least one in five patients with lung cancer had scarring, which was significantly more likely to be present in the same lobe as the tumour, suggesting a predisposition to malignancy.
\end{abstract}

S Afr Med J 2017;107(4):320-322. DOI:10.7196/SAMJ.2017.v107i4.12158

The concept of a scar carcinoma was first introduced almost 80 years ago. ${ }^{[1]}$ The presence of dense hyaline scarring in the centre of many primary peripheral lung carcinomas led to recognition of the phenomenon of a lung scar carcinoma, although it was not clear whether the relevant area of scarring preceded the carcinoma or was caused by the carcinoma. ${ }^{[1,2]}$

In recent years there has been renewed interest in scar carcinomas, also referred to as 'scar-cinomas. ${ }^{[2]}$ It has been reported that the presence of large numbers of fibroblasts and myofibroblasts is a hallmark of carcinomas. ${ }^{[3]}$ Moreover, malignant cells hijack the host wound-repair response to promote formation of the tumour stroma, which is fundamental for cancer progression. ${ }^{[3]}$ The persistence of activated mesenchymal cells is therefore critical to the pathobiology of both fibrosis and cancer. ${ }^{[2,3]}$

The Western Cape Province of South Africa (SA) has one of the highest recorded incidences of pulmonary tuberculosis (TB) in the world, and also carries a high burden of smoking-related diseases including lung cancer. ${ }^{[4]}$ However, the potential association between pulmonary scarring and lung cancer has never been described in the local population.

\section{Objective}

The main objective of this study was to assess the frequency of pulmonary scarring in all patients with lung cancer, and whether or not scarring was likely to be present in the same lobe as the lung cancer.

\section{Methods}

We retrospectively identified all patients with primary lung cancer who had presented to Tygerberg Academic Hospital and had their information captured on a prospective registry updated weekly during the combined multidisciplinary chest oncology meeting from January 2012 to December 2013. Tygerberg Hospital is a 1 380-bed tertiary facility in Cape Town, SA. It is one of two referral centres in the city and renders a tertiary service to a population of about 1.5 million with a TB incidence of approximately $1000 / 100000$ population. ${ }^{[5]} \mathrm{We}$ collected data on all patients with a known diagnosis of lung cancer and who underwent a staging computed tomography (CT) scan. Routine demographic and clinical data were collected, including smoking status (to qualify as a smoker, a patient had to have smoked at least 10 packyears per lifetime) and performance status according to the Eastern Cooperative Oncology Group (ECOG).

All patients had access to positron emission tomography-CT, bronchoscopy with endobronchial ultrasound-guided transbronchial needle aspiration with rapid onsite evaluation, transthoracic imageguided biopsy (ultrasound or CT) and related diagnostic techniques that were performed at the discretion of the treating physicians as per standard operating procedures. Patients were subsequently staged according to the 2009 International Association for the Study of Lung Cancer TNM staging system (7th edition). ${ }^{[6]}$

Pulmonary scarring (Fig. 1) was assessed by two experienced radiologists (blinded to the histological diagnosis) and categorised according to location as present in: (i) the same lobe as the primary tumour, (ii) a different lobe of the same lung, or (iii) some part of the contralateral lung; or (iv) as diffuse. Post-obstructive bronchiectasis and other changes secondary to the lung cancer itself were considered not to represent scarring.

\section{Ethics approval}

Ethics approval for this retrospective analysis was received from the Stellenbosch University Research Ethics Committee (ref. no. 


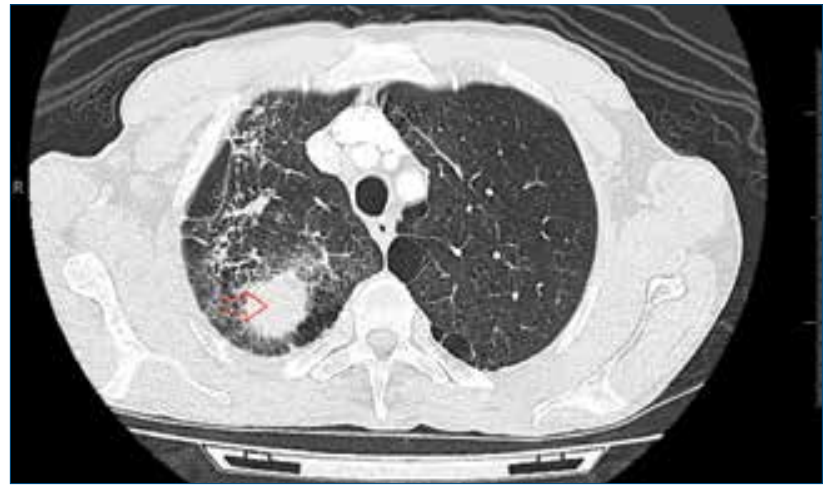

Fig. 1. An adenocarcinoma (arrow) in the background of structural lung disease (previous pulmonary TB).

S13/04/078). The application included a waiver of consent owing to the retrospective nature and anonymity of the study design.

\section{Statistical analysis}

Descriptive statistics and $\chi^{2}$ comparisons of proportional data were performed. A $p$-value $<0.05$ in a two-tailed test of proportions $\left(\chi^{2}\right)$ was considered significant.

\section{Results}

Of 508 patients with lung cancer identified, 435 had staging CT scans (mean age (standard deviation (SD)) 60.1 (10.4) years), 266 males) (Table 1). Of these, 95 patients $(21.8 \%)$ had CT evidence of pulmonary scarring. Patients with scarring were younger than those without scarring (mean 57.9 (10.2) years v. 60.7 (10.4) years; $p=0.02)$, with a male predominance in both groups $(62 / 95(65.3 \%)$ and 204/340 (60.0\%); $p=0.35)$. Cigarette smoking exposure between the groups was equal, with regard to both proportion of smokers $(85 / 95(89.5 \%)$ v. $308 / 340(90.6 \%) ; p=0.89)$ and pack-years $(27.9$ v. $31.0 ; p=0.22)$. The distribution of non-small-cell lung cancer (NSCLC) ( $n=375)$ and small-cell lung cancer (SCLC) ( $n=60)$ was comparable between the groups, as was tumour staging (Table 1).

Eighty-three of 85 patients (97.6\%) had focal scarring in the same lobe as the primary tumour. Of these, 37 (43.5\%) also had scarring involving a different lobe of the same lung, whereas only one $(1.2 \%$; $p<0.001)$ had scarring isolated to a different lobe of the same lung. Moreover, 21 patients (24.7\%) also had scarring of the opposite lung, but only one patient $(1.2 \% ; p<0.001)$ had scarring isolated to the contralateral lung. Ten patients had diffuse scarring; causes included bronchiectasis $(n=5)$, idiopathic pulmonary fibrosis $(n=4)$ and silicosis $(n=1)$.

\section{Discussion}

We found a very strong association, albeit it not a temporal association, between pulmonary scarring and lung cancer. At least one in five patients who presented with lung cancer had radiological evidence of associated scarring. Moreover, scarring was significantly more likely to be present in the same lobe as the tumour than in a different lobe, suggesting an association between scarring and lung cancer. Patients with pulmonary scarring and lung cancer were significantly younger than those without scarring, but gender, histological features, staging and smoking history were comparable between the two groups of patients.

Our study largely echoed findings of previous studies with regard to the existence of a strong anatomical relationship between a pulmonary malignancy and an associated area of fibrosis, as well as a predominance of adenocarcinomas in these malignancies. However,

\begin{tabular}{|c|c|c|c|}
\hline & Scarring present $(N=95)$ & Scarring absent $(N=340)$ & $p$-value \\
\hline Age (yr), mean (SD) & $57.9(10.2)$ & $60.7(10.4)$ & 0.02 \\
\hline \multicolumn{4}{|l|}{ Sex, $n(\%)$} \\
\hline Males & $62(65.2)$ & $204(60.0)$ & 0.35 \\
\hline \multicolumn{4}{|l|}{ Smoking status } \\
\hline Smokers, $n(\%)$ & $85(89.5)$ & $308(90.6)$ & 0.89 \\
\hline Pack-years, mean (SD) & $27.9(16.0)$ & $31.0(19.3)$ & 0.22 \\
\hline Lung cancer type, $n$ (\%) & & & 0.76 \\
\hline Adenocarcinoma & $41(43.2)$ & $137(40.3)$ & \\
\hline Squamous cell & $26(27.4)$ & $77(22.6)$ & \\
\hline Poorly differentiated & $13(13.7)$ & $67(19.7)$ & \\
\hline SCLC & $14(14.7)$ & $46(13.5)$ & \\
\hline Other & $1(1.1)$ & $12(4.7)$ & \\
\hline NSCLC staging $(N=375)^{*}, n(\%)$ & & & 0.89 \\
\hline I & $3(3.7)$ & $9(3.4)$ & \\
\hline II & $4(4.9)$ & $14(5.3)$ & \\
\hline IIIA & $3(3.7)$ & $19(7.2)$ & \\
\hline IIIB & $13(16.0)$ & $65(24.6)$ & \\
\hline IV & $58(71.6)$ & $187(70.8)$ & \\
\hline SCLC staging $(N=60), n(\%)$ & & & 1.00 \\
\hline Limited & $3(21.4)$ & $10(27.8)$ & \\
\hline Extensive & $11(78.6)$ & $36(78.2)$ & \\
\hline ECOG performance status, $n(\%)$ & & & 0.49 \\
\hline $1-2$ & $61(64.2)$ & $231(67.9)$ & \\
\hline $3-4$ & $34(36.8)$ & $109(32.1)$ & \\
\hline
\end{tabular}


we found a larger proportion of scar carcinomas in comparison with previous studies. Auerbach et al. ${ }^{[7]}$ reported autopsy findings on 1186 cases of primary lung cancer and showed that 82 cases (6.9\%) had an associated focus of pulmonary fibrosis. Raeburn and Spencer, ${ }^{[1]}$ who performed an autopsy study on 400 cases of lung cancer, found that $15(3.8 \%)$ of the cancers fitted the definition of a scar carcinoma. Freant et al. ${ }^{[8]}$ observed a $20 \%$ prevalence of pulmonary scar carcinomas in 94 surgically excised primary lung cancers. The higher proportion in our population is probably related to the high prevalence of post-tuberculous lung disease in the Western Cape. Patients with a history of pulmonary TB have been shown to display a two-fold increase in risk for the subsequent development of lung cancer. ${ }^{[9]}$

The pathogenesis of lung fibrosis is accepted to be due to the accumulation of activated reparative mesenchymal cells (myofibroblasts) and their persistent unopposed deposition of extracellular matrix (ECM). ${ }^{[10]}$ It is hypothesised that this unopposed accumulation of ECM interacts malignantly with surrounding epithelial cells by disturbing their normal cell cycles and promoting subsequent mutations. ${ }^{[11]}$

Studies evaluating fibrotic biopsies from patients with idiopathic pulmonary fibrosis (IPF) have shown a paucity of apoptosis, similar to that of malignancies, which may indicate a synonymous ability of both fibrotic and malignant tissue to evade cell death. ${ }^{[2,10]} \mathrm{A}$ possible explanation for this lack of apoptosis could be explained by overexpression of anti-apoptotic protein kinases, such as survivin. ${ }^{[12]}$ Furthermore, the process of autophagy, by which cell death occurs via the lysosomal pathway, has been shown to be impaired in IPF as well as fibroblast models. Impaired autophagy is a commonly accepted mechanism of tumourigenesis. ${ }^{[2]}$

Sustained proliferative signalling is also considered to be a hallmark of tumourigenesis, in which sustained exogenous stimuli 'hijack' the cell cycle. ${ }^{[13]}$ This overexpression of proliferative signalling networks has been shown also to be present in fibroblasts from IPF biopsies. Another accepted hallmark of malignant tissue is its ability to evade growth suppressors. Prostaglandin $\mathrm{E}_{2}\left(\mathrm{PGE}_{2}\right)$ is a cell mediator that inhibits proliferation, differentiation and collagen synthesis of normal fibroblasts as well as promoting their susceptibility to apoptosis. However, fibroblasts within IPF, similarly to malignant cells, have been shown to demonstrate less sensitivity to the effects of $\mathrm{PGE}_{2}{ }^{[2,13]}$

Our study has implications for both routine clinical care and future research. Although not specifically designed to test the hypothesis, our findings suggest that scarring (from any cause) should be viewed as a risk factor for lung cancer, and that extra vigilance is required when following up patients with existing scarring, particularly if they are smokers or have any clinical or radiological features of cancer. Prospective studies are needed to gauge the true risk associated with pulmonary scarring, particularly secondary to $\mathrm{TB}$.

\section{Study limitations}

Our study has some limitations, most notably its retrospective nature and the lack of histological confirmation of apparent scarring observed on CT scanning. Moreover, our registry was not set up to capture HIV status or a history of previous pulmonary TB. However, in the vast majority of cases with focal scarring, the CT scan was consistent with changes of previous TB.

\section{Conclusion}

At least $20 \%$ of patients with lung cancer had scarring, which was significantly more likely to be present in the same lobe as the lung cancer than elsewhere in the lungs, suggesting a predisposition to malignancy.

1. Raeburn C, Spencer H. A study of the origin and development of lung cancer. Thorax 1953;8(1):1-10. 2. Horowitz JC, Osterholzer JJ, Marazioti A, Stathopoulos GT. 'Scar-cinoma': Viewing the fibrotic lung mesenchymal cell in the context of cancer biology. Eur Respir J 2016;47(6):1842-1854. https://doi. org/10.1183/13993003.01201-2015

3. Schäfer M, Werner S. Cancer as an overhealing wound: An old hypothesis revisited. Nat Rev Mol Cell Biol 2008;9(8):628-638. https://doi.org/10.1038/nrm2455

4. Koegelenberg CFN, van der Made T, Taljaard J, Irusen E. The impact of HIV infection on the presentation of lung cancer in South Africa. S Afr Med J 2016;106(7):666-668. https://doi.org/10.7196/ SAMJ.2016.v106i7.10737

5. World Health Organization. Global Tuberculosis Report 2014. Geneva: WHO, 2014. http://apps.who . World Health Organization. Global Tuberculosis Report 2014. Geneva: WHO, 2014. http://
int/iris/bitstream/10665/137094/1/9789241564809_eng.pdf (accessed 25 November 2016).

6. Detterbeck FC, Boffa DJ, Tanoue LT. The new lung cancer staging system. Chest 2009;136(1):260-271. https://doi.org/10.1378/chest.08-0978

7. Auerbach O, Garfinkel L, Parks V. Scar cancer of the lung: Increase over a 21 year period. Cancer 1979;43(2):636-642.

8. Freant L, Joseph W, Adkins P. Scar carcinoma of the lung. Fact or fantasy? Ann Thorac Surg 1974;17(6):531-537.

9. Liang $\mathrm{H}$, Li X, Yu X, et al. Facts and fiction of the relationship between preexisting tuberculosis and lung cancer risk: A systematic review. Int J Cancer 2009;125(12):2936-2944. https://doi.org/10.1002/ijc.24636

10. Thannickal V, Henke C, Horowitz J, et al. Matrix biology of idiopathic pulmonary fibrosis: A workshop report of the national heart, lung, and blood institute. Am J Pathol 2014;184(6):1643-1651. https://doi. org/10.1016/j.ajpath.2014.02.003

11. Horowitz J, Thannickal V. Epithelial-mesenchymal interactions in pulmonary fibrosis. Semin Respir Crit Care Med 2006;27(6):600-612. https://doi.org/10.1055/s-2006-957332

12. Ajayi I, Sisson T, Higgins P, et al. X-linked inhibitor of apoptosis regulates lung fibroblast resistance to Fas-mediated apoptosis. Am J Respir Cell Mol Biol 2013;49(1):86-95. https://doi.org/10.1165/ to Fas-mediated ap

13. Grimminger F, Günther A, Vancheri C. The role of tyrosine kinases in the pathogenesis of idiopathic pulmonary fibrosis. Eur Respir J 2015;45(5):1426-1433. https://doi.org/10.1183/09031936.00149614

Accepted 1 December 2016. 